\title{
New utility of an old marker: serum low-density lipoprotein predicts histopathological response of neoadjuvant chemotherapy in locally advanced
} gastric cancer

\author{
This article was published in the following Dove Press journal: \\ OncoTargets and Therapy \\ 12 August 2016 \\ Number of times this article has been viewed
}

\author{
Ji-Chun Zhou' \\ Ju-Feng Guo ${ }^{1,2}$ \\ Rong-Yue Teng' \\ Qin-Chuan Wang' \\ Ji Wang' \\ Qun Wei ${ }^{1,3}$ \\ Zi-Duo Li ${ }^{1,4}$ \\ Jian-Guo Shen' \\ Lin-Bo Wang ${ }^{1,5}$
}

'Department of Surgical Oncology, Sir Run Run Shaw Hospital, Zhejiang University School of Medicine, ${ }^{2}$ Department of Surgical Oncology, Hangzhou First People's Hospital, Hangzhou, People's Republic of China; ${ }^{3}$ Department of International Medicine and Molecular Biology, University of Texas Southwestern Medical Center, Dallas, TX, USA;

${ }^{4}$ Dendritic Cell Biology and

Therapeutic Group, ANZAC Research Institute, University of Sydney, Sydney, NSW, Australia; ${ }^{5}$ Biomedical Research Center and Key Laboratory of Biotherapy of Zhejiang Province, Hangzhou, People's Republic of China

Correspondence: Jian-Guo Shen; Lin-Bo Wang

Department of Surgical Oncology, Sir Run Run Shaw Hospital, Zhejiang University School of Medicine, 3 East Qingchun Road, Jianggan, Hangzhou, Zhejiang 310016, People's Republic of China Email drshenjianguo@yahoo.com.cn; wanglinbo@medmail.com.cn
Background: Although the correlation between metabolic abnormality and gastric cancer has been extensively investigated, the question of whether metabolic parameters might influence the efficacy of chemotherapy in locally advanced gastric cancer is still unanswered. In our present study, we investigated the relationship between serum fasting glucose, lipid levels, and histopathological response of neoadjuvant chemotherapy (NAC) in locally advanced gastric cancers.

Patients and methods: A total of 128 patients were identified from a prospectively maintained database of patients with locally advanced gastric cancer who received NAC between July 2004 and December 2012. Histopathological response after NAC was analyzed according to Becker's tumor-regression grade. Univariate analyses and multivariable regression analyses were performed to determine the correlation between tumor size, differentiation, fasting glucose, lipid levels, and tumor histopathological response after NAC.

Results: Univariate analysis revealed that low-density lipoprotein level and total cholesterol, as well as tumor size and differentiation, correlated significantly with histopathological response. Low-density lipoprotein levels and tumor size were found to be independent predictors for histopathological response, according to multivariable regression analyses.

Conclusion: In this observational, hypothesis-generating study, serum low-density lipoprotein measurement was found to be useful in predicting chemosensitivity to locally advanced gastric cancer patients undergoing NAC. Incorporation of serum low-density lipoprotein levels into individualized treatment protocols could be considered in clinical practice.

Keywords: gastric cancer, neoadjuvant chemotherapy, low-density lipoprotein, histopathological response, predictive biomarker

\section{Introduction}

Gastric cancer is the fourth-most common cancer worldwide and the second-leading cause of global cancer deaths. The 5-year overall survival of patients diagnosed with gastric cancer is still less than $25 \%$, despite improved surgical and adjuvant treatment approaches. ${ }^{1,2}$ Recent advances in neoadjuvant chemotherapy (NAC) have significantly improved the prognosis of gastric cancer patients. ${ }^{3,4} \mathrm{NAC}$ has been proven to improve $\mathrm{R} 0$ resection rate, progression-free survival, and overall survival, without increasing the risk of perioperative morbidity and mortality. ${ }^{5}$ However, the major clinical response rate after NAC has only reached $38 \%-69.7 \%,{ }^{6,7}$ and it is possible that tumors that are potentially curable by appropriate surgery could have progressed while patients are receiving NAC, and thus they might be harmed. 
Therefore, NAC requires accurate, noninvasive techniques to assess short-term therapeutic response and avoid the morbidity and costs of ineffective therapy, which would markedly facilitate the use of NAC and improve prognosis for gastric cancer patients. The current standard method for discriminating chemotherapeutic responders from nonresponders is based on postoperative tumor-specimen pathologic analysis, which is applicable only in a postoperative setting and cannot be used for either the preoperative design of personalized treatment or during the course of NAC administration.

Previous studies have also revealed that the conventional anatomic imaging modalities (computed tomography, magnetic resonance imaging, and endoscopic ultrasonography) for assessing tumor volumetry lack reliability for predicting response to chemotherapy, ${ }^{8,9}$ which might be limited in interpreting imaging findings due to chemotherapy-induced edema and fibrosis. The recently developed technology of functional imaging appears to be a promising alterative for monitoring chemotherapeutic effects in gastric tumors. Ott et al demonstrated that response to preoperative chemotherapy could be predicted by ${ }^{18} \mathrm{~F}$-fluorodeoxyglucose positronemission tomography during the course of therapy. ${ }^{10}$ Most recently, Ang et al demonstrated that double contrastenhanced ultrasonography may represent an innovative tool for more accurately predicting histopathological response. ${ }^{11}$ However, the sensitivity and specificity of these modalities still do not meet clinical requirements, which impedes their general acceptance for routine clinical use. Furthermore, these imaging technologies are restricted from widespread use due to technical complexity and high cost, rendering them frequently not amenable to use in smaller, underfunded clinics, especially in rural areas.

Numerous preclinical studies have shown that metabolic disturbance interferes with important signaling pathways that might serve as drivers of tumor initiation and progression. Serum metabolic parameters could be promising biomarkers to delineate subpopulations susceptible to adverse events, and thus may play a potential role in predicting chemosensitivity for cancer patients prior to commencement of chemotherapy. Accumulating epidemiological evidence has linked metabolic syndrome as well as its individual components with an increased risk of colorectal cancer, ${ }^{12}$ breast cancer, ${ }^{13,14}$ endometrial cancer. ${ }^{15}$ Of considerable potential interest is the fact that levels of low-density lipoprotein (LDL)-derived lipids have proven to be a predictive biomarker for capecitabineassociated toxicity in colon cancer patients. ${ }^{16}$ Another study demonstrated that patients with metastatic cancer and concurrent metabolic syndrome showed increased resistance to standard treatments, as measured by treatment-response assessment. ${ }^{17}$ Moreover, another study identified fasting serum glucose and triglyceride concentrations as clinically useful pharmacodynamic markers of mTOR inhibition. ${ }^{18}$

For gastric cancer, it is believed that serum lipids and glucose levels might also influence cancer risk and prognosis. The positive or negative associations between gastric cancer and serum metabolic parameters (including glucose, total cholesterol, triglycerides, and LDL cholesterol) ${ }^{19-24}$ have been extensively investigated. However, these studies looked only at the association between the serum metabolic parameters and impaired tumor initiation, not the remission of preexisting tumors after treatment (the latter being the more clinically relevant situation). Recently, Wei et al demonstrated that metabolic syndrome was associated with better tumor-cell differentiation and was an independent predictor for better survival in a subgroup of patients with early stage gastric cancer. ${ }^{25}$ There have been virtually no studies regarding the relevance of these metabolic syndrome factors to chemotherapy efficacy in gastric cancer, despite the considerable overlap between these hormones in terms of their receptors and intracellular signaling pathways. In this study, we attempted to clarify the value of pretreatment serum fasting glucose and lipids for predicting gastric cancer chemosensitivity in a neoadjuvant setting.

\section{Patients and methods}

\section{Patients and tumor characteristics}

This study included 128 consecutive locally advanced gastric cancer patients (clinically staged as T2 and above or with lymph-node metastasis) who had undergone NAC followed by surgical resection at the Surgical Oncology Department, Sir Run Run Shaw Hospital, Zhejiang University School of Medicine, between July 2004 and December 2012. The study protocol was approved by the institutional review board of Sir Run Run Shaw hospital. Written informed consent according to institutional guidelines was obtained from all patients.

Twelve patients were excluded: pretreatment serum glucose or lipid levels were unavailable in eleven cases, and one patient had undergone laparotomy and histopathological response could not be assessed. Clinical staging was based on the International Union Against Cancer and American Joint Committee on Cancer TNM system on gastric cancer. Information on patient demographics and tumor characteristics, such as age, sex, stage, presence of regional lymphnode or distant metastases, histological subtype, tumor grade according to World Health Organization classification, 
Table I Reference range of fasting serum glucose and lipid profiles

\begin{tabular}{lll}
\hline Metabolic variables & $\begin{array}{l}\text { Normal (optimal), } \\
\mathbf{m g} / \mathbf{d L}\end{array}$ & $\begin{array}{l}\text { High, } \\
\mathbf{m g} / \mathbf{d L}\end{array}$ \\
\hline Glucose & $70-100$ & $\geq 120$ \\
Triglycerides & $<150$ & $\geq 150$ \\
Total cholesterol & $<200$ & $\geq 200$ \\
Low-density lipoprotein & $<100$ & $\geq 100$ \\
Very low-density lipoprotein & $<30$ & $\geq 30$ \\
High-density lipoprotein & $>60$ & $\leq 60$ \\
\hline
\end{tabular}

serum glucose, lipid level, and body mass index (BMI), was obtained from our prospectively collected and computerized institutional databases.

Pretreatment blood samples were taken to measure fasting glucose, complete lipid profile, including total cholesterol, LDL, high-density lipoprotein, very LDL, and triglycerides. These pretreatment parameters were categorized into two groups according to normal reference ranges (summarized in Table 1). BMI was calculated from patients' measured height and weight. All measurements were taken prior to NAC administration according to fixed protocols. Patients underwent a treatment assessment scan every two cycles of NAC, and standard Response Evaluation Criteria in Solid Tumors criteria (version 1.1) were used to assess response to treatment and determine subsequent strategy. ${ }^{26}$

\section{Histopathological response evaluation}

The Becker score was implemented to evaluate histopathological response, based on an estimation of the percentage of residual tumor tissue in relation to the macroscopically identifiable tumor bed. ${ }^{27}$ Tumor regression was classified into three grades: grade 1, complete or subtotal regression ( $<10 \%$ residual tumor per tumor bed); grade 2 , partial tumor regression ( $10 \%-50 \%$ residual tumor per tumor bed); and grade 3 , minimal or no tumor regression $(>50 \%$ residual tumor per tumor bed). All patients with grade 1 or 2 regression were classified as responders, while grade 3 was defined as a pathologic nonresponse. The Becker system uses the percentage of viable tumor cells as a measure of response, an approach that appears to be more easily and reproducibly identifiable than the use of the degree of fibrosis and tumor necrosis employed by the other two scoring systems. ${ }^{28-30}$

\section{Statistical analysis}

Continuous variables are reported as mean \pm standard deviation. Fisher's exact or $\chi^{2}$ tests were used to assess correlations of discrete covariate distributions and histopathological response groups. Student's $t$-test (for parametric distribution) or the Mann-Whitney $U$ test (for nonparametric distribution or distributions not meeting criteria for normality and homogeneity of variance) was applied to compare continuous variables between two subgroups. Multivariate logistic regression models were performed to assess the association between histopathological response and potentially predictive parameters adjusted for different clinicopathological covariates (ie, age, sex, tumor stage and grade, and metastatic status). SPSS 19.0 (IBM Corporation, Armonk, NY, USA) was used for statistical assessment. In all tests, two-sided $P<0.05$ was considered significant.

\section{Results \\ Clinicopathological characteristics and pathologic tumor response after neoadjuvant chemotherapy}

Our patient population with measurable clinicopathological information consisted of $88(75.9 \%)$ males and $28(24.1 \%)$ females with a mean (median) age of $59.03 \pm 10.378$ years (range: $28-78$ years). The median BMI for all patients was $21.72 \pm 2.97 \mathrm{~kg} / \mathrm{m}^{2}$. Pathologic tumor response after NAC was evaluated based on Becker's score. Tumor regression was grade 1 in 16 patients (13.8\%), grade 2 in 56 patients (48.3\%), and grade 3 in 44 patients (37.9\%). Therefore, 72 (62.1\%) patients were categorized as pathologic responders and $44(37.9 \%)$ categorized as pathologic nonresponders (summarized in Table 2). Details of patient management and clinicopathological features are summarized in Table 3.

\section{Clinicopathological variables predicting pathologic tumor response}

For univariate analysis, clinicopathological parameters and treatment variables - age, sex, BMI, clinical $\mathrm{T}$ and $\mathrm{N}$ staging, tumor location, differentiation, macroscopic type, tumor size, chemotherapy regimens, serum fasting glucose, and lipid profile - were documented to determine correlation with tumor regression. Univariate predictors of

Table 2 Pathological response after neoadjuvant chemotherapy according to Becker score

\begin{tabular}{llll}
\hline Response & Grade & Frequency & Percentage \\
\hline Pathological response & Grade I ( $<10 \%$ residual tumor per tumor bed $)$ & 16 & 13.8 \\
& Grade 2 (10\%-50\% residual tumor per tumor bed) & 56 & 48.3 \\
Pathological nonresponse & Grade 3 ( $>50 \%$ residual tumor per tumor bed $)$ & 44 & 37.9 \\
\hline
\end{tabular}


Table 3 Univariate analysis to identify predictors of tumor regression in patients with neoadjuvant chemotherapy

\begin{tabular}{|c|c|c|c|}
\hline \multirow[t]{2}{*}{ Characteristic } & \multicolumn{2}{|c|}{ Tumor-regression grade } & \multirow[t]{2}{*}{$P$-value } \\
\hline & Grades I and 2, n & Grade 3, n & \\
\hline \multicolumn{3}{|l|}{ Age, years } & $0.77 \mathrm{I}$ \\
\hline$<60$ & 34 & 22 & \\
\hline$\geq 60$ & 38 & 22 & \\
\hline \multicolumn{3}{|l|}{ Sex } & 0.537 \\
\hline Male & 56 & 32 & \\
\hline Female & 16 & 12 & \\
\hline \multicolumn{3}{|l|}{ BMI, kg/m² } & 0.377 \\
\hline \multirow[t]{2}{*}{$<20$} & 19 & 15 & \\
\hline & 53 & 29 & \\
\hline \multicolumn{3}{|l|}{ Tumor location } & 0.713 \\
\hline Upper body & 12 & 7 & \\
\hline Middle body & 19 & 8 & \\
\hline Lower body & 38 & 26 & \\
\hline Diffuse type & 3 & 3 & \\
\hline \multicolumn{3}{|c|}{ Tumor differentiation } & 0.013 \\
\hline Differentiated & 29 & 8 & \\
\hline Undifferentiated & 43 & 36 & \\
\hline Tumor size, cm & $4.02 \pm 2.26$ & $6.15 \pm 2.9$ & $>0.001$ \\
\hline \multicolumn{3}{|l|}{ Borrmann type } & 0.488 \\
\hline I & 6 & 3 & \\
\hline II & 23 & 9 & \\
\hline III & 37 & 26 & \\
\hline IV & 6 & 6 & \\
\hline \multicolumn{3}{|c|}{ Clinical T classification } & 0.111 \\
\hline cT2 & 10 & 1 & \\
\hline cT3 & 52 & 37 & \\
\hline cT4 & 10 & 6 & \\
\hline \multicolumn{3}{|c|}{ Clinical $\mathbf{N}$ classification } & $0.37 \mid$ \\
\hline $\mathrm{cN}^{-}$ & 22 & 17 & \\
\hline $\mathrm{cN}^{+}$ & 50 & 27 & \\
\hline \multicolumn{3}{|c|}{ Chemotherapy regimen } & 0.582 \\
\hline FOLFOX & 51 & 35 & \\
\hline ECF or modified ECF & 14 & 6 & \\
\hline Others ${ }^{\mathrm{a}}$ & 7 & 3 & \\
\hline \multicolumn{3}{|c|}{ Chemotherapy cycles } & 0.985 \\
\hline 2 & 19 & 11 & \\
\hline 3 & 18 & 11 & \\
\hline$\geq 4$ & 35 & 22 & \\
\hline \multicolumn{3}{|l|}{ Surgical type } & 0.132 \\
\hline Radical & 62 & 33 & \\
\hline Palliative & 10 & 11 & \\
\hline \multicolumn{3}{|l|}{ Glucose } & 0.933 \\
\hline $70-100 \mathrm{mg} / \mathrm{dL}$ & 48 & 29 & \\
\hline$\geq 120 \mathrm{mg} / \mathrm{dL}$ & 24 & 15 & \\
\hline \multicolumn{3}{|l|}{ Triglycerides } & 0.103 \\
\hline$<150 \mathrm{mg} / \mathrm{dL}$ & 55 & 39 & \\
\hline$\geq 150 \mathrm{mg} / \mathrm{dL}$ & 17 & 5 & \\
\hline \multicolumn{3}{|l|}{ Total cholesterol } & 0.008 \\
\hline$<200 \mathrm{mg} / \mathrm{dL}$ & 59 & 43 & \\
\hline$\geq 200 \mathrm{mg} / \mathrm{dL}$ & 13 & 1 & \\
\hline \multicolumn{3}{|c|}{ Low-density lipoprotein } & 0.008 \\
\hline$<100 \mathrm{mg} / \mathrm{dL}$ & 48 & 39 & \\
\hline$\geq 100 \mathrm{mg} / \mathrm{dL}$ & 24 & 5 & \\
\hline
\end{tabular}

Table 3 (Continued)

\begin{tabular}{|c|c|c|c|}
\hline \multirow[t]{2}{*}{ Characteristic } & \multicolumn{2}{|c|}{ Tumor-regression grade } & \multirow[t]{2}{*}{$P$-value } \\
\hline & Grades $I$ and 2, n & Grade 3, n & \\
\hline \multicolumn{3}{|c|}{ Very Low-density lipoprotein } & 0.692 \\
\hline$<30 \mathrm{mg} / \mathrm{dL}$ & 50 & 29 & \\
\hline$\geq 30 \mathrm{mg} / \mathrm{dL}$ & 22 & 15 & \\
\hline \multicolumn{3}{|c|}{ High-density lipoprotein } & 0.443 \\
\hline$>60 \mathrm{mg} / \mathrm{dL}$ & 10 & 4 & \\
\hline$\leq 60 \mathrm{mg} / \mathrm{dL}$ & 62 & 40 & \\
\hline
\end{tabular}

Note: aRadiochemotherapy and paclitaxel-based.

Abbreviations: BMI, body mass index; FOLFOX, folinic acid + fluorouracil + oxaliplatin; ECF, epirubicin + cisplatin + fluorouracil.

tumor regression included tumor differentiation $(P=0.013)$, tumor size $(P<0.001)$, total cholesterol $(P=0.008)$, and LDL ( $P=0.008$ ) (summarized in Table 2).

For multivariate analysis, a logistic regression model was utilized to evaluate the relationship between each dependent variable. Variables studied on univariate analysis were entered into the logistic regression model. Multivariable analysis yielded two independent predictors of tumor regression: tumor size $(P=0.024)$ and LDL $(P=0.001)$. Patients with smaller tumors and higher LDL levels tended to benefit more from NAC (summarized in Table 4).

\section{Discussion}

In this observational, hypothesis-generating study using NAC as an in vivo chemosensitivity test, we demonstrated that serum LDL and tumor size may predict the likelihood of benefit from NAC in patients with locally advanced gastric cancer. Utilizing the Becker score ${ }^{27}$ to evaluate post-NAC histopathological response, we demonstrated that patients with higher LDL levels and smaller tumors tended to be more sensitive to NAC. While the biological mechanism for such a relationship has yet to be determined, to our knowledge this is the first study to demonstrate that serum LDL levels may be correlated with histopathological response from NAC.

Gastric cancer represents an enormous clinical and economic burden to health care systems worldwide, particularly in Asian countries, such as Japan, the People's Republic of China, and South Korea. The high disease incidence and

Table 4 Multivariate logistic analysis to identify predictors of tumor regression in patients with neoadjuvant chemotherapy

\begin{tabular}{llll}
\hline Variables & Odds ratio & $\mathbf{9 5 \%} \mathbf{C l}$ & $P$-value \\
\hline $\begin{array}{l}\text { Low-density lipoprotein } \\
(<100 \mathrm{mg} / \mathrm{dL} \text { vs } \geq 100 \mathrm{mg} / \mathrm{dL})\end{array}$ & 3.187 & $1.164-8.728$ & 0.024 \\
Tumor size & 1.636 & $1.213-2.207$ & 0.001 \\
\hline
\end{tabular}


poor outcomes continue to make gastric cancer a topic of active clinical and basic scientific research. NAC has become a standard-care regimen for patients with locally advanced gastric cancer. Indeed, NAC has been proven to improve R0 resection rate, progression-free survival, and overall survival, without affecting perioperative morbidity and mortality in locally advanced gastric cancer. ${ }^{3-5}$ However, the selection of appropriate patients who will benefit from NAC remains an important clinical issue. Our ability to predict the chemosensitivity of NAC properly has not kept pace with its increasing importance in cancer treatment. Ineffective chemotherapy exposes patients to potentially avoidable toxicity and delays surgical treatment, during which time there is potential for disease progression.

A technique for measuring response to NAC prior to initiation of medical treatment would greatly aid clinicians in designing precision cancer treatments. Many types of predictive tools have been investigated, but widespread implementation is hindered by lack of reliability ${ }^{8,9}$ and accessibility, as well as the complexity of the modalities. ${ }^{10,31-33}$ There is a clear and unmet need for predictive tools or guidance that could provide information to support treatment decision making in these clinical situations.

Most recently, Liu et al demonstrated that the levels of Let7i in pretreatment tumor tissue might be a potential tissue marker for the prediction of chemotherapeutic sensitivity in patients with locally advanced gastric cancer. ${ }^{34}$ Moreover, our previous study also showed that Lin 28 expression was correlated with pathologic tumor response in locally advanced gastric cancer patients undergoing NAC. ${ }^{35}$ Although this was a well-designed study, measurement of tissue Let $7 \mathrm{i}$ is still not validated or readily available in the clinical setting. Serum biomarkers offer the opportunity for more objective and reproducible measurements prior to administration of NAC, and so the utility of LDL as a worldwide well-standardized and validated parameter should not be underestimated. Our present study suggested that LDL, an inexpensive and readily available validated laboratory parameter, may be a clinically relevant pharmacodynamic marker of response to NAC for locally advanced gastric cancer.

There continues to be insufficient evidence to warrant serum LDL for predictive use in therapeutic decision making. However, the incorporation of this and other molecular prediction tools for more specifically characterizing patients should certainly be assessed in prospective gastric cancer NAC studies. Such a strategy should not only identify unresponsive cases in a timely manner, in order to initiate individualized treatment options, but also provide alternative end points for identifying nonresponders and prognostic parameters for assessing individual prognosis. ${ }^{36}$ In our current study, which included the predictive value of LDL for NAC efficacy, we also found that high LDL was correlated with better prognosis (data not shown).

Although the correlation between serum LDL and cancer risk has been extensively investigated, a potential role for LDL in predicting chemosensitivity has not been reported. LDL cholesterol appear to be associated with increased risk for gastric dysplasia, ${ }^{24}$ which is believed to be the penultimate stage of gastric carcinogenesis. ${ }^{37}$ In addition, high serum cholesterol levels have been proven to be an independent protective factor for developing gastric cancer, a fact which is particularly true for early gastric cancer and in particular intestinal-type gastric cancer. ${ }^{21}$ Serum total-cholesterol levels were inversely associated with risk of total cancer in males, with strong inverse associations in particular with gastric cancer. ${ }^{22}$ Along with circulating lipids, an experimental study has shown that dietary lipids may induce local inflammation in the alimentary tract, a phenomenon that is thought to enhance the development of gastrointestinal malignancies. ${ }^{38}$

This study expands on the existing literature by demonstrating that in addition to their roles in gastric cancer development, serum LDL levels can be used to predict the chemosensitivity of NAC in locally advanced gastric cancers. Furthermore, this study adds to the growing body of literature suggesting that serum metabolic parameters, including lipids and glucose, may be useful to predict the efficacy of cancer treatment. For example, metabolic syndrome had been implicated in the increasing resistance to standard treatments in metastatic breast cancer patients. ${ }^{17}$ Moreover, another study revealed that changes in fasting serum glucose and triglyceride concentrations might be independent predictive biomarkers for mTOR inhibition. ${ }^{18}$

To date, the precise molecular mechanism by which LDL levels might affect chemotherapy response is not known. Based on the phenomena observed in the present study, as well as a review of currently available literature, we propose four potential mechanisms that might explain, at least partially, why pretreatment high serum LDL is correlated with histopathological response: 1) since the efficacy of chemotherapy is suspected to be strongly influenced by a variety of host factors responsible for the agent's metabolism, high LDL levels may influence the host's ability to metabolize antineoplastic agents, resulting in different histopathological response; 2) since many chemotherapeutic agents 
attack tumor cells in the replicative phase, high LDL might be synergistic; 3) LDL receptors are upregulated in many neoplastic cell lines, ${ }^{39,40}$ and uptake of extracellular cholesterol through the LDL receptor increases, ${ }^{41}$ which could result in increasing toxic-drug uptake in tumor cells; importantly, the LDL-receptor endocytic pathway can be used as a gateway for delivery of anticancer drugs to tumor tissues; ${ }^{42} 4$ ) circulating microRNAs are relatively stable in plasma, and are a new class of disease biomarkers; research has demonstrated that high-density lipoprotein transports endogenous microRNAs and delivers them to tumor cells with functional targeting capabilities; ${ }^{43}$ LDL might also participate in intercellular communication concerning transportation and delivery of microRNAs, which have already been well established as critical players in chemotherapy resistance. ${ }^{44}$

Undoubtedly, much work has yet to be done to tease out the molecular underpinnings behind this relationship, but such findings could have a major impact not only on which patients with advanced gastric cancer should receive NAC but also how this information may be interpreted and acted on by both research participants and clinicians. The possibility that circulating factors (in this study, LDL) may be associated with chemotherapy response is appealing, because it could support and promote further research on other predictive biomarkers for response to chemotherapy, as well as drugresistance mechanisms, in patients with cancers treated with similar types of chemotherapy.

As a retrospective observational study with relatively small sample size, this study was limited by lack of complete information about the use of cholesterol-inhibiting drugs, and might have been confounded by the use of different NAC regimens and cycles. Therefore, the present correlation between LDL and tumor regression should be interpreted cautiously until a large-scale, well-designed prospective study is available.

\section{Conclusion}

Though requiring further confirmation, this primary evidence indicates that LDL may be incorporated as part of a personalized approach to predictions of efficacy from NAC in locally advanced gastric cancer. Assuming future research supports this finding, it is reasonable to propose that the optimal utilization of NAC may be at least partially based on serum LDL levels.

\section{Acknowledgments}

We thank Amanda N Kallen MD for her special critical review. This study was supported by grants from the National Natural Science Foundation of China (81101659/H1609,
81101659), Natural Science Foundation of Zhejiang Province (Y2110073), Science and Health Care Foundation of Zhejiang Province (2011KYA086), Program for Innovative Research Team in Zhejiang Province (2010R50046), and the Specialized Research Fund for the Doctoral Program of Higher Education of China (20100101110127).

\section{Disclosure}

The authors report no conflicts of interest in this work.

\section{References}

1. Siegel R, Naishadham D, Jemal A. Cancer statistics, 2012. CA Cancer J Clin. 2012;62:10-29.

2. Ferlay J, Shin HR, Bray F, Forman D, Mathers C, Parkin DM. Estimates of worldwide burden of cancer in 2008: GLOBOCAN 2008. Int J Cancer. 2010;127:2893-2917.

3. Cunningham D, Allum WH, Stenning SP, et al. Perioperative chemotherapy versus surgery alone for resectable gastroesophageal cancer. N Engl J Med. 2006;355:11-20.

4. Schuhmacher C, Gretschel S, Lordick F, et al. Neoadjuvant chemotherapy compared with surgery alone for locally advanced cancer of the stomach and cardia: European Organisation for Research and Treatment of Cancer randomized trial 40954. J Clin Oncol. 2010;28:5210-5218.

5. Ge L, Wang HJ, Yin D, et al. Effectiveness of 5-flurouracil-based neoadjuvant chemotherapy in locally-advanced gastric/gastroesophageal cancer: a meta-analysis. World J Gastroenterol. 2012;18:7384-7393.

6. De Vita F, Orditura M, Matano E, et al. A phase II study of biweekly oxaliplatin plus infusional 5-fluorouracil and folinic acid (FOLFOX-4) as first-line treatment of advanced gastric cancer patients. Br J Cancer. 2005;92:1644-1649.

7. Li ZY, Koh CE, Bu ZD, et al. Neoadjuvant chemotherapy with FOLFOX: improved outcomes in Chinese patients with locally advanced gastric cancer. J Surg Oncol. 2012;105:793-799.

8. Mallery S, DeCamp M, Bueno R, et al. Pretreatment staging by endoscopic ultrasonography does not predict complete response to neoadjuvant chemoradiation in patients with esophageal carcinoma. Cancer. 1999;86:764-769.

9. Brown WA, Thomas J, Gotley D, et al. Use of oesophagogastroscopy to assess the response of oesophageal carcinoma to neoadjuvant therapy. Br J Surg. 2004;91:199-204.

10. Ott K, Fink U, Becker K, et al. Prediction of response to preoperative chemotherapy in gastric carcinoma by metabolic imaging: results of a prospective trial. J Clin Oncol. 2003;21:4604-4610.

11. Ang J, Hu L, Huang PT, et al. Contrast-enhanced ultrasonography assessment of gastric cancer response to neoadjuvant chemotherapy. World J Gastroenterol. 2012;18:7026-7032.

12. Mutoh M, Akasu T, Takahashi M, et al. Possible involvement of hyperlipidemia in increasing risk of colorectal tumor development in human familial adenomatous polyposis. Jpn J Clin Oncol. 2006;36:166-171.

13. Furberg AS, Veierød MB, Wilsgaard T, Bernstein L, Thune I. Serum high-density lipoprotein cholesterol, metabolic profile, and breast cancer risk. J Natl Cancer Inst. 2004;96:1152-60.

14. Healy LA, Ryan AM, Carroll P, et al. Metabolic syndrome, central obesity and insulin resistance are associated with adverse pathological features in postmenopausal breast cancer. Clin Oncol ( $R$ Coll Radiol). 2010;22:281-8.

15. Furberg AS, Thune I. Metabolic abnormalities (hypertension, hyperglycemia and overweight), lifestyle (high energy intake and physical inactivity) and endometrial cancer risk in a Norwegian cohort. Int J Cancer. 2003;104:669-676.

16. Backshall A, Sharma R, Clarke SJ, Keun HC. Pharmacometabonomic profiling as a predictor of toxicity in patients with inoperable colorectal cancer treated with capecitabine. Clin Cancer Res. 2011;17:3019-3028. 
17. Stebbing J, Sharma A, North B, et al. A metabolic phenotyping approach to understanding relationships between metabolic syndrome and breast tumour responses to chemotherapy. Ann Oncol. 2012;23:860-866.

18. Nallari AS, Karrison T, Rosner GL, et al. Fasting glucose and triglycerides as biomarkers of mTOR inhibition, evidence of a categorical response. J Clin Oncol. 2010;28:3091.

19. Tseng CH. Diabetes conveys a higher risk of gastric cancer mortality despite an age-standardised decreasing trend in the general population in Taiwan. Gut. 2011;60:774-779.

20. Ge Z, Ben Q, Qian J, Wang Y, Li Y. Diabetes mellitus and risk of gastric cancer: a systematic review and meta-analysis of observational studies. Eur J Gastroenterol Hepatol. 2011;23:1127-1135.

21. Asano K, Kubo M, Yonemoto K, et al. Impact of serum total cholesterol on the incidence of gastric cancer in a population-based prospective study: the Hisayama study. Int J Cancer. 2008;122:909-914.

22. Iso H, Ikeda A, Inoue M, Sato S, Tsugane S. Serum cholesterol levels in relation to the incidence of cancer: the JPHC study cohorts. Int $J$ Cancer. 2009; 125:2679-2686.

23. Kitayama J, Tabuchi M, Tsurita G, Ishikawa M, Otani K, Nagawa H. Adiposity and gastrointestinal malignancy. Digestion. 2009;79 Suppl 1: 26-32.

24. Jung MK, Jeon SW, Cho CM, et al. Hyperglycaemia, hypercholesterolaemia and the risk for developing gastric dysplasia. Dig Liver Dis. 2008;40:361-365.

25. Wei X, Qiu M, Lin H, et al. Patients with old age or proximal tumors benefit from metabolic syndrome in early stage gastric cancer. PLoS One. 2014;9:e89965.

26. Eisenhauer EA, Therasse P, Bogaerts J, et al. New response evaluation criteria in solid tumours: revised RECIST guideline (version 1.1) Eur J Cancer. 2009;45:228-247.

27. Becker K, Mueller JD, Schulmacher C, et al. Histomorphology and grading of regression in gastric carcinoma treated with neoadjuvant chemotherapy. Cancer. 2003;98:1521-1530.

28. Mandard AM, Dalibard F, Mandard JC, et al. Pathologic assessment of tumor regression after preoperative chemoradiotherapy of esophageal carcinoma: clinicopathologic correlations. Cancer. 1994;73: 2680-2686.

29. Ninomiya Y, Yanagisawa A, Kato Y, Kitagawa T, Ishihara S, Nakajima T Histological indications of a favorable prognosis with far-advanced gastric carcinomas after preoperative chemotherapy. J Cancer Res Clin Oncol. 1999;125:699-706.

30. Mirza A, Naveed A, Hayes S, et al. Assessment of histopathological response in gastric and gastro-oesophageal junction adenocarcinoma following neoadjuvant chemotherapy: which scoring system to use? ISRN Pathol. 2012;2012:519351.
31. Wang LB, Teng RY, Jiang ZN, et al. Clinicopathologic variables predicting tumor response to neoadjuvant chemotherapy in patients with locally advanced gastric cancer. J Surg Oncol. 2012;105:293-296.

32. Marcus CD, Ladam-Marcus V, Cucu C, Bouché O, Lucas L, Hoeffel C. Imaging techniques to evaluate the response to treatment in oncology: current standards and perspectives. Crit Rev Oncol Hematol. 2009; 72:217-238.

33. Lorenzen S, von Gall C, Stange A, et al. Sequential FDG-PET and induction chemotherapy in locally advanced adenocarcinoma of the oesophago-gastric junction (AEG): the Heidelberg imaging program in cancer of the oesophago-gastric junction during neoadjuvant treatment - HICON trial. BMC Cancer. 2011;11:266.

34. Liu K, Qian T, Tang L, Wang J, Yang H, Ren J. Decreased expression of microRNA let-7i and its association with chemotherapeutic response in human gastric cancer. World J Surg Oncol. 2012;10:225.

35. Teng RY, Zhou JC, Jiang ZN, et al. The relationship between Lin 28 and the chemotherapy response of gastric cancer. Onco Targets Ther. 2013;6:1341-1345.

36. Langer R, Ott K, Feith M, Lordick F, Siewert JR, Becker K. Prognostic significance of histopathological tumor regression after neoadjuvant chemotherapy in esophageal adenocarcinomas. Modern Pathol. 2009;22:1555-1563.

37. de Vries AC, van Grieken NC, Looman CW, et al. Gastric cancer risk in patients with premalignant gastric lesions: a nationwide cohort study in the Netherlands. Gastroenterology. 2008;134:945-952.

38. Ji Y, Sakata Y, Tso P. Nutrient-induced inflammation in the intestine. Curr Opin Clin Nutr Metab Care. 2011;14:315-321.

39. Vitols S, Peterson C, Larsson O, Holm P, Aberg B. Elevated uptake of low density lipoproteins by human lung cancer tissue in vivo. Cancer Res. 1992;52:6244-6247.

40. Ho YK, Smith RG, Brown MS, Goldstein JL. Low-density lipoprotein (LDL) receptor activity in human acute myelogenous leukemia cells. Blood. 1978;52:1099-1114.

41. Tatidis L, Masquelier M, Vitols S. Elevated uptake of low density lipoprotein by drug resistant human leukemic cell lines. Biochem Pharmacol. 2002;63:2169-2180.

42. Rudling MJ, Collins VP, Peterson CO. Delivery of aclacinomycin A to human glioma cells in vitro by the low-density lipoprotein pathway. Cancer Res. 1983;43:4600-4605.

43. Vickers KC, Palmisano BT, Shoucri BM, Shamburek RD, Remaley AT. MicroRNAs are transported in plasma and delivered to recipient cells by high-density lipoproteins. Nat Cell Biol. 2011;13:423-433.

44. Blower PE, Chung JH, Verducci JS, et al. MicroRNAs modulate the chemosensitivity of tumor cells. Mol Cancer Ther. 2008;7:1-9.
OncoTargets and Therapy

\section{Publish your work in this journal}

OncoTargets and Therapy is an international, peer-reviewed, open access journal focusing on the pathological basis of all cancers, potential targets for therapy and treatment protocols employed to improve the management of cancer patients. The journal also focuses on the impact of management programs and new therapeutic agents and protocols on

\section{Dovepress}

patient perspectives such as quality of life, adherence and satisfaction. The manuscript management system is completely online and includes a very quick and fair peer-review system, which is all easy to use. Visit http://www.dovepress.com/testimonials.php to read real quotes from published authors. 